\title{
Leucemias agudas y síndromes mielodisplásicos secundarios al tratamiento oncológico
}

\author{
R. DÍAZ BEVERIDGE, J. APARICIO URTASUN
}

Servicio de Oncología Médica. Hospital Universitario La Fe. Valencia

THERAPY-RELATED ACUTE LEUKAEMIA AND MYELODISPLASTIC SYNDROME

\section{RESUMEN}

Las neoplasias hematológicas secundarias representan una complicación grave del tratamiento oncológico. Se desconoce su incidencia real dada la heterogeneidad de los tumores primarios, su pronóstico y su tratamiento. Suelen manifestarse como leucemias agudas y síndromes mielodisplásicos y, entre ellos, destacan dos entidades nosológicas con características clínicas y citogenéticas propias: la asociada al empleo de alquilantes y aquella secundaria al uso de epipodofilotoxinas. El diagnóstico se basa en la sospecha clínica, las alteraciones morfológicas y el estudio citogenético. Su pronóstico es uniformemente desfavorable. La quimioterapia convencional tiene un objetivo paliativo y sólo el trasplante alogénico permite la curación en un número limitado de casos. Por ello deben optimizarse las pautas terapéuticas en aquellas neoplasias primarias con posibilidad de obtener largas supervivencias y mantener a los pacientes en programas de seguimiento prolongado.

PALABRAS CLAVE: Leucemia aguda secundaria. Síndrome mielodisplásico. Alquilantes. Epipodofilotoxinas. Radioterapia.

\begin{abstract}
Secondary hematological malignancies represent a severe complication of cancer treatment. Their real incidence is unknown because of the heterogeneity of primary tumors, their therapies, and their prognosis. The usual presentation is an acute leukemia or myelodisplastic syndrome. Two different diseases have been described with particular clinical and cytogenetic features, namely the one associated with alkylating drugs and that related to epipodophylotoxins. Diagnosis is based on clinical suspicion, morphological alterations and cytogenetic studies. Prognosis is uniformly dismal. Conventional chemotherapy is mainly palliative, whereas allogenic transplantation allows the cure of a small percentage of cases. Thus, potential curative therapies for solid tumors should be optimized and patients maintained in long-term surveillance programs.
\end{abstract}

KEY WORDS: Secondary acute leukemia. Myelodisplastic syndrome. Alkylating agents. Epipodophilotoxins. Radiotherapy.

Díaz, Beveridge R, Aparicio Urtasun J. Leucemias agudas y síndromes mielodisplásicos secundarios al tratamiento oncológico. An Med Interna (Madrid) 2003; 20: 257-268.

\section{INTRODUCCIÓN}

La inducción de neoplasias secundarias al tratamiento quimioterápico y radioterápico es una complicación infrecuente pero grave del tratamiento oncológico. Dentro de estas, desde hace más de 40 años, se conocen como entidades clínicas bien diferenciadas los síndromes mielodisplásicos (SMD) y las leucemias agudas mieloides (LAM) secundarias, asociadas tradicionalmente al uso de agentes alquilantes. Se observan con más frecuencia en los supervivientes a largo plazo de la enfermedad de Hodgkin y de los linfomas no hodgkinianos (1). A su vez, en los últimos años, se ha demostrado la existencia de otro subtipo de leucemia aguda secundaria, descrita originaria- mente en niños con leucemia aguda linfoblástica (LAL) que se trataron con epipodofilotoxinas, no precedida habitualmente de SMD y de características clínicas, biológicas y citogenéticas diferentes a la LAM secundaria "clásica" $(2,3)$. En ambos casos se postula que el mecanismo de leucemogénesis está asociado al daño del ADN de las células hematopoyéticas de la médula ósea por dichos agentes quimioterápicos. A pesar de las diferencias entre ambos subtipos, el pronóstico es uniformemente desfavorable y la única terapia curativa en la actualidad es el trasplante alogénico de médula ósea, con tasas de curación a los 5 años del 25-30\% (4,5).

La importancia de estas LAM secundarias es probable que vaya en aumento en los próximos años debido al uso cada vez

Trabajo aceptado: 7 de noviembre de 2003 
más frecuente de agentes genotóxicos, el empleo de estos fármacos en distintas combinaciones y en dosis cada vez mayores, y por el aumento de la supervivencia media de los pacientes oncológicos $(6,7)$. Dadas las escasas perspectivas de curación con los tratamientos actuales, es obvio que se necesitan técnicas de prevención y de diagnóstico precoz de dichas neoplasias; de especial interés sería reconocer los cambios inducidos en el material genético de las células por los agentes quimioterápicos y los mecanismos de reparación por parte de la célula, así como reconocer aquellos pacientes que, por razones genéticas o adquiridas, son más susceptibles a los efectos leucemogénicos de la quimioterapia, lo que permitiría la modificación consecuente del tratamiento (7).

El propósito de esta revisión, por lo tanto, es describir las características clínicas, biológicas y citogenéticas de estas neoplasias secundarias, así como su relación con algunos de los tumores primarios más frecuentes; se esboza a su vez el pronóstico de estos procesos, incidiendo en las distintas armas terapéuticas disponibles en la actualidad.

\section{CARACTERÍSTICAS GENERALES}

Las LAM son neoplasias hematológicas caracterizadas por la proliferación incontrolada de blastos de estirpe mieloide en la médula ósea y los tejidos periféricos (6). Se diferencian de acuerdo a las características citológicas, inmunofenotípicas y citogenéticas en siete subtipos de acuerdo a la clasificación
FAB (French-American-British) (Tabla I) (8-9). Los SMD, en cambio, son procesos dishematopoyéticos de la médula ósea caracterizados por la alteración en la maduración y diferenciación de las líneas celulares hematopoyéticas (con afectación de una, dos o de las tres líneas celulares sanguíneas) y, en algunos casos, por la presencia de blastos en la médula ósea, sin cumplir criterios definitorios de leucemia aguda (10). Tradicionalmente estos procesos se han clasificado en 5 subtipos de la FAB de acuerdo a los hallazgos en médula ósea y en sangre periférica (Tabla II) (11). Ambos procesos se pueden clasificar en primarios (sin factor etiológico asociado) o secundarios (desencadenados por agentes industriales, como el benzeno, o por agentes quimioterápicos) $(12,13)$. La transformación a leucemia aguda en los SMD primarios se da en el $10 \%$ de casos y es más frecuente en los subtipos más agresivos (anemia refractaria con exceso de blastos con/sin transformación); en los SMD secundarios dicha transformación es mucho más frecuente, alcanzando porcentajes del 55\% al $84 \%$ en algunas series $(1,14,15)$.

La incidencia de ambos procesos no está claramente definida, aunque se considera que un $10 \%$ de las LAM son probablemente secundarias (16). Estas diferencias epidemiológicas se deben, por una parte, a la ausencia de características diagnósticas definitorias de ambos procesos (el diagnóstico se basa en los antecedentes clínicos y en algunas características citogenéticas) y, por otra parte, a la dificultad de relacionar la neoplasia con el tratamiento oncológico previo (por las grandes variaciones en el uso de los distintos agentes quimioterá-

TABLA I

CLASIFICACIÓ N FAB DE LAS LEUCEM IAS M IELO IDES AGUDAS

\begin{tabular}{|c|c|c|c|c|}
\hline & M orfología & Histoquímica (1) & Inmunofenotipo (2) & Citogenética \\
\hline MO & Indiferenciada & $\begin{array}{l}\text { M PO }+<3 \% \text {; PAS } \\
\text { y esterasa - }\end{array}$ & $\begin{array}{l}\text { HLA-DR, CD13 } \\
\text { CD33, CD34 +; CD7 Y TdT +/- }\end{array}$ & $\begin{array}{l}\text { 11q13; cambios complejos en } \\
5 \text { o 7; t }(9,22) \text { ocasionalmente }\end{array}$ \\
\hline M 1 & M ieloide & $\begin{array}{l}\text { M PO }+<3 \% ; \text { PAS } \\
\text { y esterasa - }\end{array}$ & Similar a M 0 excepto CD15 +/ - & $-5,-7,-17$, del $3 p,+21,+8$ \\
\hline M 2 & $\begin{array}{l}\text { M ieloide con } \\
\text { diferenciación }\end{array}$ & $\begin{array}{l}\text { M PO }>10 \% ; \text { PAS } \\
\text { y esterasa - }\end{array}$ & $\begin{array}{l}\text { HLA-DR +; CD13, CD33 +; } \\
\text { CD34 +/-; CD15 + }\end{array}$ & $\begin{array}{l}\mathrm{t}(8 ; 21) ; \text { del } 3 p \text { o inv(3); } \\
-5,-7, \mathrm{t}(6,9),+8\end{array}$ \\
\hline M3 & Promielocítica & $\begin{array}{l}\text { M PO ++; PAS y } \\
\text { esterasa - }\end{array}$ & $\begin{array}{l}\text { HLA-DR -; CD13, CD15, CD33, } \\
\text { CD34 +/- }\end{array}$ & $\mathrm{t}(15 ; 17)$ \\
\hline M 4 & M ielomonocítica & $\begin{array}{l}\text { M PO y esterasa +; } \\
\text { PAS - }\end{array}$ & $\begin{array}{l}\text { HLA-DR, CD14, CD15 +/-; } \\
\text { CD4 débil +; CD34 +/-; } \\
\text { CD33 > CD13; CD11b + }\end{array}$ & $\begin{array}{l}\ln v(16) 0-16 q ; t(8,21) \text { a veces, } \\
-5,-7,+8\end{array}$ \\
\hline M 5 & M onocítica & $\begin{array}{l}\text { M PO -; PAS y } \\
\text { esterasa + }\end{array}$ & & $\mathrm{t}(9 ; 11)(\mathrm{p} 21 ; \mathrm{p} 23),+8$ \\
\hline M 6 & Eritroleucemia & $\begin{array}{l}\text { PAS ++; M PO y } \\
\text { esterasa - }\end{array}$ & $\begin{array}{l}\text { HLA-DR, CD13, CD33 +/-; } \\
\text { glicoforina A ++ }\end{array}$ & $\begin{array}{l}-7 \text { o del }(7 q) \text { y/0 }-50 \text { del } \\
-3,+8\end{array}$ \\
\hline M 7 & M egacarioblástica & $\begin{array}{l}\text { PAS +/ -; M PO y } \\
\text { esterasa - }\end{array}$ & $\begin{array}{l}\text { HLA-DR , CD34 +; CD33 +/-; } \\
\text { CD41, CD61 + glicoproteína } \\
\text { plaquetaria + }\end{array}$ & $\begin{array}{l}\mathrm{t}(12 ; 21) \text { en } 20-25 \% \\
\text { hiperploidia; }+8,+21\end{array}$ \\
\hline
\end{tabular}

(1) M PO : mielopeoxidasa; PAS: ácido periódico de Schiff.

(2) TdT, HLA-DR, CD34: anticuerpos monoclonales frente a células precursoras; CD13, CD33, CD15: anticuerpos monoclonales frente a células mieloides; CD11 y CD14: anticuerpos monoclonales frente a células monocitarias. 
picos, sus dosis acumuladas, el uso o no asociado de radioterapia e incluso el pronóstico del tumor primario).

\section{CLÍNICA Y DIAGNÓSTICO}

Ambos procesos provocan sintomatología aproximadamente en el 75 a $100 \%$ de pacientes, con clínica predominante de astenia y debilidad generalizada debida a la anemia prácticamente constante que presentan estos pacientes $(100 \%$ de casos); son frecuentes las manifestaciones hemorrágicas leves, como epistaxis o petequias $(50-60 \%)(1,15,17)$. La presencia de fiebre se da en el 40 a $60 \%$ de pacientes, pero la documentación microbiológica de la infección se da en menos del $50 \%$ de éstos. Entre las alteraciones analíticas, como es lógico, la anemia y la trombocitopenia son prácticamente constantes, con hemoglobinas medias menores de 10 $\mathrm{g} / \mathrm{dl}$ y cifras de plaquetas medias menores de 100.000/ul; la presencia de leucopenia es más rara, con cifras medias de neutrófilos menores de 500/ul en solo el $10 \%$ de pacientes $(10,15,17)$. La visión en sangre periférica de las tres series sanguíneas, sobre todo en los SMD, demuestra alteraciones morfológicas secundarias al proceso dishematopoyético en la médula ósea (Tabla III) $(10,18)$. La médula ósea presenta una apariencia variable, siendo lo más frecuente una médula ósea hipercelular (19) con mielodisplasia generalizada llamativa; son menos frecuentes las médulas óseas hipocelulares (25$50 \%$ de casos) y normocelulares (10-25\%).

Un problema importante en el diagnóstico de los SMD y las LAM secundarias es que rara vez cumplen estrictamente los criterios diagnósticos de la FAB. Los patrones más frecuentes en casos de SMD son la anemia refractaria con exceso de blastos (AREB) y la anemia refractaria con exceso de blastos en transformación (AREBt), aunque muchas veces no se establece el diagnóstico debido a que el porcentaje de blastos en médula ósea es menor del $5 \%(15,19)$; por otra parte, en el caso de la anemia refractaria simple y de la anemia refractaria sideroblástica, el grado de disgranulopoyesis y dismegacariopoyesis es mayor del que cabría esperar.

En el caso de las LAM secundarias, están representados la mayor parte de los subtipos de la $\mathrm{FAB}$, con predominio de M1-M2 y M5-M6, aunque su diagnóstico es dificil por la frecuente afectación de las tres series. Aunque la inmensa mayoría de los casos corresponden a LAM, se han descrito esporádicamente casos de LAL y de leucemia mieloide crónica asociadas al tratamiento quimioterápico (20).

En resumen, el diagnóstico tanto de la LAM secundaria como del SMD secundario al tratamiento quimioterápico se establece mediante el análisis de la sangre periférica y de la médula ósea en el contexto clínico adecuado (por ejemplo, anemia refractaria no filiada en un paciente oncológico). No existen criterios diagnóstico definitivos de ambos procesos, aunque la frecuente afectación de las tres series, la alteración mielodisplásica llamativa y, fundamentalmente, unas alteraciones citogenéticas específicas orientan hacia su diagnóstico.

\section{LEUCEMIA MIELOIDE AGUDA SECUNDARIA A AGENTES ALQUILANTES}

La LAM secundaria al uso de agentes alquilantes es la forma de presentación clásica de estos procesos y se describió originariamente en supervivientes a largo plazo de la enfermedad de Hodgkin tratados con el protocolo MOPP (21). Esta neoplasia presenta un fenotipo clínico y morfológico característico: típicamente presenta una fase mielodisplásica previa que culmina en una LAM con un subtipo de la FAB poco frecuente (M6 y M7 con mielofibrosis acompañante). El pico de incidencia de

TABLA II

CLASIFICACIÓ N FAB DE LOS SÍNDRO M ES M IELO DISPLÁSICOS

\begin{tabular}{|c|c|c|c|c|c|}
\hline & $\begin{array}{l}\text { Anemia } \\
\text { refractaria }\end{array}$ & $\begin{array}{c}\text { Anemia } \\
\text { refractaria } \\
\text { sideroblástica }\end{array}$ & $\begin{array}{c}\text { Anemia } \\
\text { refractaria con } \\
\text { exceso de blastos }\end{array}$ & $\begin{array}{l}\text { Anemia } \\
\text { refractaria con } \\
\text { exceso de blastos } \\
\text { en transformación }\end{array}$ & $\begin{array}{l}\text { Leucemia } \\
\text { mielomonocítica } \\
\text { crónica }\end{array}$ \\
\hline $\begin{array}{l}\text { Disgranulopoyesis } \\
\text { Exceso de blastos } \\
\text { Bastones Auer }\end{array}$ & $\begin{array}{c}\text { Escasa } \\
<5 \% \\
\text { Negativos }\end{array}$ & $\begin{array}{c}\text { Escasa } \\
<5 \% \\
\text { Negativos }\end{array}$ & $\begin{array}{l}\text { Importante } \\
5-20 \% \\
\text { Negativos }\end{array}$ & $\begin{array}{c}\text { Importante } \\
20-30 \% \\
\text { Definitorios (1) }\end{array}$ & $\begin{array}{c}\text { Variable } \\
1-20 \% \\
\text { Negativos }\end{array}$ \\
\hline $\begin{array}{l}\text { Diseritropoyesis } \\
\text { Sideroblastos en anillo }\end{array}$ & $\begin{array}{l}\text { Frecuente } \\
\text { Infrecuente }\end{array}$ & $\begin{array}{l}\text { Característica } \\
>15 \% \text { en M.0. }\end{array}$ & $\begin{array}{l}\text { Frecuente } \\
\text { Frecuente }\end{array}$ & $\begin{array}{l}\text { Frecuente } \\
\text { Frecuente }\end{array}$ & $\begin{array}{l}\text { Variable } \\
\text { Variable }\end{array}$ \\
\hline Dismegacariopoyesis & Infrecuente & Infrecuente & Frecuente & Frecuente & Variable \\
\hline $\begin{array}{l}\text { Monocitos en sangre } \\
\text { periférica (x } 10 \text { 9/L) }\end{array}$ & $<1 \%$ & $<1 \%$ & $<1 \%$ & Variable & $>1 \%$ \\
\hline $\begin{array}{l}\text { \% de progresión } \\
\text { leucémica }\end{array}$ & 12 & 8 & 44 & 66 & 14 \\
\hline $\begin{array}{l}\text { Supervivencia media } \\
\text { meses }\end{array}$ & 32 & 42 & 12 & 5 & 20 \\
\hline
\end{tabular}

(1) La presencia de bastones de Auer en las células de la médula ósea definen el subtipo AREB-T, aunque el porcentaje de blastos fuera menor del $20 \%$. 
TABLA III

HALLAZGOS EN SANGRE PERIFÉRICA Y EN M ÉDULA Ó SEA EN LOS SÍNDRO M ES M IELO DISPLÁSICO S

\begin{tabular}{llll}
\hline & Serie roja & Serie blanca & Serie plaquetaria \\
\hline Sangre periférica & Anemia, anisocitosis, punteado & Formas inmaduras con blastos & Formas gigantes, con degranulación, \\
& basófilo, microcitos, cuerpos de & circulantes, neutrófilos & trombocitopenia, megacarioblastos \\
& Howell-jolly, poikilocitosis, & hipogranulares, monocitosis, & y micromegacariocitos, fragmentos \\
& eritroblastos circulantes & basofilia, formas seudo-Pelger- & de megacarioblastos circulantes \\
& & Huert &
\end{tabular}

Médula ósea

M icroscopio

electrónico

Hallazgos

funcionales

\begin{abstract}
Eritroblastosis, diseritropoyesis, hiperplasia eritroide con rasgos megaloblásticos, sideroblastos anillados
\end{abstract}

\section{Hiposegmentación nuclear, bastones de Auer, hipogranulación citoplasmática, maduración megaloblastoide}

Hiperplasia megacariocítica, formas gigantes, micromegacariocitos, megacarioblastos, formas mononucleares

Formación de gránulos primarios y secundarios disminuida, gránulos anormales, cromatina anómala ("clumping") repliegues de membrana celular redundante
Aumento de hemoglobina fetal, síntesis de cadenas de globinas aberrante, pérdida de antígenos sanguíneos

\author{
$\downarrow F A L, \downarrow$ mieloproxidasa, \\ $\uparrow$ muraminidasa, quimiotaxis y \\ fagocitosis alterada, antígenos de \\ superficie anómalos, $\downarrow$ actividad
}

\section{Núcleos hiperlobulados, nucleos hipolobulados, gránulos gigantes, membranas difuminadas}
Agregación alterada, déficit de tromboxano A2, defecto Bernard- Soulier-like bactericida

da 4 a 6 años después del tratamiento quimioterápico del tumor primario, aunque la latencia puede variar desde 12 meses hasta incluso 15-20 años (7). En estos procesos son frecuentes las alteraciones en los cromosomas 5 y 7 , en forma de pérdidas cromosómicas $(22,23)$. La anormalidad más frecuente es la monosomía del cromosoma 7 (39\% de casos), seguida de la deleción 5q (18\%) y de la monosomía del cromosoma 5 (14\%); en algunas series se observan alteraciones combinadas de ambos cromosomas hasta en un $90 \%$ de casos.

Una zona crítica en su patogénesis es el brazo largo del cromosoma 7 que se encuentra afectado en la mayor parte de casos, ya sea en forma de monosomía, de pérdida del brazo largo o incluso de traslocaciones descompensadas con pérdida de dicho brazo, describiéndose alteraciones cromosómicas del tipo $\mathrm{t}(1,7)(\mathrm{p} 11 ; \mathrm{q} 11), \mathrm{t}(5,7)(\mathrm{q} 11.2 ; \mathrm{p} 11.2)$ y $\mathrm{t}(7,17)(\mathrm{p} 11 ; \mathrm{p} 11)$ [24]. A pesar de dichas alteraciones, no se ha podido implicar ningún gen en dicha patogénesis.

El cromosoma 5 codifica una serie de genes clave que dirigen la hematopoyesis y pueden jugar un papel en el comienzo y en la progresión del clon leucémico. La región 5q31-33 del brazo largo del cromosoma 5 codifica por lo menos 9 genes que influyen en la diferenciación y en el crecimiento de la célula hematopoyética (7). Esta región contiene los genes codificadores del factor estimulante de colonias granulocitomacrofágicas (GM-GSF) y de las interleukinas 3, 4, 5 y 9; también contiene el gen regulador del factor de interferón 1 (IRF1), el activador transcripcional de los genes de los interferones y el gen de respuesta de crecimiento precoz 1 (ERG1), necesario para la diferenciación de la línea monocito-macrofágica. Otros genes incluyen al oncogen FMS (que codifica el receptor celular del factor estimulante de colonias de macrófagos, o CSF1) y el gen CDC25C (que codifica la fosfatasa activadora de la ciclinkinasa $\mathrm{CDC} 2$ ).
Los agentes alquilantes y compuestos relacionados son fármacos que contienen en su estructura grupos químicos capaces de formar enlaces covalentes con sustancias nucleofílicas en las células. El N7 de la guanina probablemente sea la diana molecular fundamental en la alquilación del ADN, aunque probablemente también sean alquilados el N1 y N3 de la adenosina y el N3 de la citosina. Al ser habitualmente moléculas con dos grupos alquilantes, los fármacos son capaces de provocar entrecruzamientos dentro de la misma cadena de ADN y entre cadenas distintas, interfiriendo en la transcripción y, sobre todo, en la replicación del ADN. Incluyen fármacos tan importantes en el tratamiento quimioterápico como las mostazas nitrogenadas, las nitrosureas, el busulfán y los agentes relacionados, cisplatino y carboplatino, con átomos de platino en su estructura molecular.

La mayor parte de estudios de la leucemogenicidad de los agentes alquilantes se basan en estudios de pacientes tratados de enfermedad de Hodgkin o que recibieron quimioterapia adyuvante por un cáncer de mama. Los agentes alquilantes con efectos leucemogénicos demostrados incluyen la mecloretamina, clorambucilo, ciclofosfamida, melfalán, semustina, lomustina, carmustina, prednimustina, busulfán, dihidroxibusulfán y el alquilante no clásico procarbazina $(1,25)$. En la actualidad es dudoso el poder leucemogénico del cisplatino (26).

La determinación de la distinta leucemogenicidad de los agentes alquilantes en solitario es difícil de establecer debido a que raramente se utilizan dichos agentes en monoterapia y/o sin la adición de radioterapia. A pesar de dichas dificultades metodológicas, parecen demostrados algunos datos:

-El uso de melfalán para el tratamiento del cáncer de ovario se asocia con un riesgo de sufrir un proceso leucémico a los 10 años del $11,2 \%$ en comparación con el riesgo del $5,4 \%$ en pacientes tratados con ciclofosfamida (27). Se han 
observado datos similares en pacientes con mieloma múltiple en tratamiento con melfalán-prednisona, no obervándose un riesgo aumentado cuando se tratan con ciclofosfamida (28).

-El busulfán es claramente más leucemogénico que la ciclofosfamida en pacientes tratados con ambos fármacos para el cáncer de pulmón (29).

- En general, parece demostrado que la ciclofosfamida es menos leucemogénica que el melfalán, mecloretamina, clorambucil, lomustina y tiotepa. Aunque históricamente se ha considerado que la procarbazina es más leucemogénica que las mostazas nitrogenadas, distintos estudios han demostrado resultados conflictivos; por ejemplo, en tumores germinales, la procarbazina no está asociada a un riesgo aumentado de leucemias agudas (30).

- Pocos estudios han podido demostrar la leucemogenicidad del cisplatino; únicamente en el cáncer de ovario se ha podido observar cierto aumento del riesgo, aunque mucho menor que con los agentes quimioterápicos utilizados previamente, como el melfalán $(26,27)$.

A pesar de las dificultades metodológicas en diferenciar entre la dosis total acumulada de agente alquilante, la intensidad de dosis y la duración total del tratamiento, parece demostrado que es la dosis total acumulada de agente alquilante el principal factor responsable en la inducción de LAM secundarias $(25,31)$. Es probable, a su vez, que el empleo conjunto de dos agentes alquilantes tenga efecto aditivo en la leucemogénesis $(27,28)$. Esta relación con la dosis total acumulada se ha demostrado para la ciclofosfamida, clorambucil, melfalán y dihidroxibusulfán. No existen pruebas que justifiquen la relación entre la forma de administración de la quimioterapia (intravenosa, oral, intermitente...) para igualdad de dosis total del agente alquilante (28).

La asociación entre la radioterapia y la quimioterapia con agentes alquilantes no presenta un riesgo aumentado de leucemogénesis con las técnicas actuales de irradiación de volúmenes pequeños; este riesgo aumentado sí se ha descrito en pacientes de linfoma no hodgkiniano tratados con agentes alquilantes asociados a técnicas de irradiación raramente utilizadas en la actualidad (como la irradiación corporal total y la irradiación hemicorporal total, que implican la el tratamiento de superficies amplias del organismo con dosis bajas de radioterapia) (31-33). Esta discordancia parece estar relacionada con el diferente efecto sobre el material genético de las distintas dosis de irradiación, favoreciéndose la mutagénesis en las células hematopoyéticas de la médula ósea tratadas con dosis bajas de irradiación, mientras que la lisis celular se favorece con dosis elevadas.

El riesgo de desarrollar LAM tras el tratamiento con agentes alquilantes es máximo tras un periodo de latencia de 4 a 6 años, presentándose la mayor parte de casos en los 10 años posteriores al tratamiento primario para ir disminuyendo progresivamente tras ese periodo $(25,34)$. Este hecho contrasta con el riesgo de tumores sólidos como segundas neoplasias que aumenta progresivamente después de los 10 años del tratamiento del tumor primario; este riesgo aumentado de segundas neoplasias sólidas es achacable a la radioterapia, sin haberse podido demostrar un papel etiológico de la quimioterapia en la tumorogénesis.

En resumen, los SMD y la LAM secundaria al uso de agentes alquilantes están relacionados de forma más o menos directa con la dosis total acumulada de agentes alquilantes utilizada, comportándose como un efecto secundario dosisdependiente; dicha capacidad leucemogénica varía entre los distintos alquilantes, siendo máxima para agentes como el melfalán y el busulfán y prácticamente nula para el cisplatino. La radioterapia actualmente tiene poco papel en el desarrollo de dichos síndromes, especialmente con las técnicas actuales de irradiación de volúmenes pequeños con dosis altas de irradiación.

\section{LEUCEMIA MIELOIDE AGUDA SECUNDARIA A EPIPODOFILOTOXINAS}

La introducción de las epipodofilotoxinas (etopósido y tenipósido) en el arsenal terapéutico de los años 80 para el tratamiento de la LAL infantil se vio acompañada unos años después por la aparición de una LAM secundaria, de características diferentes a la leucemia inducida por alquilantes, hasta en un $10 \%$ de pacientes $(2,16,35)$ (Tabla IV). Esta neoplasia presenta un periodo de latencia corto (1 a 3 años de media), una presentación abrupta y sin fase mielodisplásica previa, con predominio de los subtipos de la FAB M4-M5. Como alteraciones cromosómicas características, estas neoplasias no suelen presentar alteraciones de los cromosomas 5 y 7 , pero sí traslocaciones compensadas en las bandas $11 \mathrm{q} 23$ y $21 \mathrm{q} 22$. Especialmente importante es la traslocación compensada en la banda 11q23 (36-37), que afecta al gen MLL y es análogo del gen de diferenciación embrionaria del insecto Drosophila dudrex; se han descrito hasta 21 traslocaciones diferentes en dicha banda. A pesar de ello, el mecanismo de acción del gen MLL en humanos no está establecido en la actualidad.

Las epipodofilotoxinas son fármacos citotóxicos que actúan sobre la topoisomerasa II intranuclear impidiendo la correcta reparación del ADN. Esta leucemia secundaria se vio asociada a la administración de dosis elevadas de etopósido (hasta 9,2 a $19 \mathrm{~g} / \mathrm{m}^{2}$ ), administradas de forma intermitente con dosis intensivas y de forma semanal o quincenal. En la actua-

TABLA IV

CARACTERÍSTICAS CLÍNICAS DIFEREN CIALES DE LAS LEUCEM IAS M IELO IDES AGUDAS SECUNDARIAS

\begin{tabular}{lll}
\hline & LM A secundaria a alquilantes & LM A secundaria a epipodofilotoxinas \\
\hline Latencia (años) & $4-7$ años & $1-3$ años \\
Incidencia & 1 a $>20 \%$ & $2-12 \%$ \\
Citogenética & Del $(5 q)$, del $(7 q),-5,-7$ & Translocaciones (en 11q23) \\
Presentación & M ielodisplasia previa & Abrupta, sin mielodisplasia \\
Fenotipo & M 6 y M 7 (mielofibrosis) & M 5 y M 6 \\
Supervivencia a largo plazo & Escasa & Escasa \\
\hline
\end{tabular}


lidad, y fundamentalmente para el tratamiento de tumores sólidos en adultos, no se alcanzan dosis tan elevadas (habitualmente $<5 \mathrm{~g} / \mathrm{m}^{2}$ ) y el fármaco se administra en forma de ciclos continuos de cinco días (16); con esta forma de administración se ha observado una importante disminución del riesgo de leucemogénesis hasta casi equipararse a la de la población general. De esta forma, se puede deducir que:

-El determinante fundamental del riesgo de leucemogénesis en el tratamiento con epipodofilotoxinas viene dado por la forma de administración, siendo máximo para formas de administración intermitente semanal o quincenal. Las epipodofilotoxinas son agentes ciclo-específicos y es probable que el riesgo de inducción de leucemias dependa del modo de administración del fármaco, como se ha demostrado en distintos estudios en animales (38-39).

- Las dosis totales acumuladas tienen menor importancia que en las leucemias inducidas por agentes alquilantes, no observándose diferencias significativas entre las distintas dosis, siempre que la dosis total sea menor de $5 \mathrm{~g} / \mathrm{m}^{2}$ (16).

Un problema importante de futuro es la posible relación sinérgica entre las epipodofilotoxinas y los agentes alquilantes a altas dosis, usados especialmente para el tratamiento de tumores infantiles como el sarcoma de Ewing, en la inducción de leucemias secundarias; el uso cada vez más frecuentes de dichos protocolos intensivos de quimioterapia permitirá saber si dicha relación existe.

Las antraciclinas son fármacos citotóxicos que, entre sus distintos mecanismos de acción, inhiben la topoisomerasa II de distinta forma que las epipodofilotoxinas ; una posibilidad teórica es la inducción de leucemias similares a las producidas por las epipodofilotoxinas. El uso de dichos fármacos, habitualmente combinados con los agentes alquilantes y con la radioterapia, hace difícil valorar si dicho riesgo existe. Actualmente se considera que, si lo poseen, presentan aisladamente poco poder leucemogénico (40). El paclitaxel, sin embargo, y de forma poco clara, parece ser responsable de un pequeño número de leucemias secundarias similares a las inducidas por epipodofilotoxinas y asociadas a la inversión del cromosoma 16 como alteración característica (7). El pequeño número de casos hace difícil valorar cuáles son los factores predisponentes para esta inducción.

\section{TUMORES PRIMARIOS}

A continuación se describen las peculiaridades que presentan las LAM y SMD secundarios en relación con los principales tumores primarios en que se han detectado.

\section{ENFERMEDAD DE HODGKIN}

La elevada probabilidad de curación de la enfermedad de Hodgkin en la actualidad y la edad juvenil de los pacientes han permitido una valoración adecuada del riesgo de segundas neoplasias a largo plazo en este grupo de enfermos $(21,25,33)$. De forma consistente se ha descrito un aumento del riesgo de LAM secundarias al uso de quimioterapia y un aumento del riesgo de tumores sólidos secundarios al tratamiento radioterápico.

Se observa, en general, un riesgo relativo (RR) aumentado para la LAM secundaria (RR 70), comparado con un RR de
12 para el linfoma no hodgkiniano, de 7 a 12 para los tumores óseos y de 4 para los tumores pulmonares. A pesar de ello, dada la baja incidencia tanto de las LAM como de los linfomas no hodgkinianos en la población general, un RR alto se traducirá habitualmente en un riesgo acumulativo real bajo, comparado con el riesgo acumulativo real elevado de los tumores sólidos, dada la elevada frecuencia de esas neoplasias en la población general (41).

La LAM secundaria en la enfermedad de Hodgkin es el prototipo de leucemia aguda inducida por agentes alquilantes. A diferencia de otras neoplasias primarias donde no se puede descartar que exista un nexo común entre la neoplasia primaria y la leucemia aguda (como en el cáncer de mama), el riesgo leucemogénico se debe aquí con toda probabilidad al uso de agentes alquilantes en la quimioterapia utilizada en su tratamiento.

La introducción del protocolo MOPP (mecloretamina, vincristina, procarbazina y prednisona) está asociada a un franco aumento del riesgo leucemogénico; dicho riesgo está claramente relacionado con el número de ciclos y fundamentalmente con la dosis total acumulada de agentes alquilantes, especialmente de mecloretamina $(25,42)$. El riesgo asociado a 12 ciclos es aproximadamente de 3 a 5 veces mayor que el riesgo asociado a 6 ciclos, mientras que el RR asociado a dosis totales acumulativas de mecloretamina mayores de $110 \mathrm{mg}$ es de 60 a 80 veces más alto que en los pacientes tratados con radioterapia exclusivamente (25). El riesgo leucemogénico de la procarbazina, aunque tradicionalmente descrito como mayor que el de la mecloretamina, no ha podido demostrarse en distintos estudios, ofreciendo resultados inconsistentes (30).

La sustitución del esquema MOPP por la combinación $\mathrm{ABVD}$ ha supuesto una importante reducción del riesgo de inducción de leucemias, con tasas de RR acumulado a los 15 años de $0.7 \%$ (comparado con un $9.5 \%$ en los pacientes tratados con MOPP) $(25,43)$; incluso en pacientes tratados con regímenes combinados MOPP-ABVD, el RR a los 15 años disminuye hasta $6.4 \%$, probablemente debido a la disminución de la dosis de agentes alquilantes en el protocolo MOPP (34).

La adición de radioterapia, especialmente a las dosis y al volumen de irradiación utilizado en la enfermedad de Hodgkin, no está asociada a un aumento del riesgo leucemogénico comparado con el uso de quimioterapia aislada . La evidencia actual en la literatura médica no sugiere que exista un papel de la radioterapia en la inducción de estas neoplasias $(1,21)$.

El factor predisponente del huésped más estudiado es la edad, describiéndose tradicionalmente más riesgo en los pacientes con más edad; sin embargo, estudios con tasas de incidencia ajustadas por edad no han podido demostrar esta incidencia aumentada (44). No existe tampoco clara relación con el sexo del paciente. El papel de la esplenectomía es dudoso, variando el RR desde 1,3 hasta 14 (44). De forma interesante, los parámetros biológicos de daño previo a la médula ósea en el momento del inicio de la quimioterapia y durante el tratamiento (como la trombocitopenia) sí parecen indicar un aumento del riesgo de inducción de leucemias, con RR en algunos estudios hasta de 5 (25). La utilidad clínica de estas determinaciones está aún por aclarar.

\section{LINFOMA NO HODGKINIANO}

La inducción de segundas neoplasias en enfermos con linfoma está menos estudiada que en pacientes con enfermedad 
de Hodgkin, dada la edad más avanzada y el peor pronóstico inicial en estos pacientes. A su vez, dado que en la evolución de estos procesos es frecuente la transformación leucémica hacia LAL, el diagnóstico de LAM secundaria debe establecerse con claridad. El riesgo en este grupo de enfermos está aumentado de forma discreta (RR de 4,8) (45) y es especialmente frecuente en aquellos pacientes tratados con dosis elevadas de agentes alquilantes y con protocolos de radioterapia que incluyen irradiación considerable de la médula ósea $(46,47)$.

El riesgo de LAM secundaria está asociado, al igual que en la enfermedad de Hodgkin, con el uso de agentes alquilantes, especialmente la prednimustina (RR 13,4), con los protocolos de tipo MOPP (RR 12,6) y con el clorambucilo a dosis acumulativas superiores de $1.300 \mathrm{mg}$ (48). Es interesante que el uso de ciclofosfamida está asociado con un RR bajo de 1.8 $\mathrm{y}$, para dosis totales acumulativas menores de 12,5 gramos, no existe relación con la dosis total, lo que demuestra el escaso poder leucemogénico de la ciclofosfamida, sobre todo con las dosis utilizadas en la actualidad $(32,48)$.

De forma paradójica, el uso de técnicas de radioterapia en forma de dosis bajas sobre volúmenes amplios de médula ósea, como la irradiación corporal total y la irradiación hemicorporal total (escasamente utilizadas hoy en día) está asociado con un riesgo muy elevado de inducción de leucemias (RR de hasta 50 a 100), especialmente si se asocia a quimioterapia $(46,47)$. El uso de quimioterapia junto con técnicas de irradiación más limitadas no está asociada con un aumento del riesgo comparado con la quimioterapia aisladamente.

\section{CÁNCER DE OVARIO}

El empleo de agentes alquilantes en el cáncer de ovario está claramente establecido y se centra actualmente en el cisplatino, habitualmente combinado, para el tratamiento de la enfermedad metastática (26). El cáncer de ovario es uno de los pocos tumores donde se ha descrito un riesgo leucemogénico asociado al uso de cisplatino (RR de 4), aunque significativamente menor al riesgo asociado con el agente utilizado previamente, el melfalán (RR 20,8) (26). Esta asociación significativa entre el cisplatino y la inducción de LAM secundarias en el cáncer de ovario, a diferencia de otros tumores, no tiene una explicación clara, aunque no se puede descartar un nexo etiológico común entre ambas neoplasias.

Las leucemias agudas secundarias en las pacientes con cáncer de ovario cumplen las características típicas de las leucemias inducidas por agentes alquilantes (periodo de latencia relativamente largo, 4-6 años; fase mielodisplásica previa y alteraciones citogenéticas características a nivel de los cromosomas 5 y 7) (49). El efecto sinérgico entre ambos fármacos, melfalán y cisplatino, se asocia a un mayor riesgo (RR 21,5) comparado con el uso de ambos fármacos aisladamente (26). La radioterapia aislada no está asociado a un riesgo significativo (RR 0,4), aunque hay un discreto aumento de la incidencia con la asociación de cisplatino y radioterapia (RR 8,1) (26). El RR para ambos fármacos aumenta progresivamente con el aumento de las dosis totales acumuladas, mientras que la forma de administración, ya sea intravenosa u oral, a igualdad de dosis no presenta variación del riesgo.

A pesar del efecto leucemogénico del cisplatino en monoterapia, los beneficios de dicha quimioterapia en el tratamien- to de la enfermedad diseminada en la supervivencia superan ampliamente el riesgo de emplearlo. Sin embargo, una preocupación para el futuro es el uso cada vez más frecuente de combinaciones de quimioterapia que incluyen el cisplatino y otros agentes quimioterápicos con efectos leucemogénicos demostrados, como la adriamicina y la ciclofosfamida, por lo que es probable que la incidencia vaya en aumento (26).

\section{TUMORES GERMINALES}

La introducción de la quimioterapia con cisplatino en los años 70 para el tratamiento de los tumores testiculares diseminados supuso un auténtico paso adelante en la curación de estos pacientes. Los estudios posteriores sobre segundas neoplasias (50-52) demostraron un RR para las leucemias agudas secundarias discretamente aumentado (RR 5,1). Sin embargo, es posible que este riesgo sólo sea parcialmente secundario al tratamiento; por una parte, es probable que exista un nexo común etiológico entre ambas neoplasias y se ha descrito en tumores germinales mediastínicos la asociación específica con una LAM de estirpe megacarioblástica, con periodo de latencia corto y asociada a una alteración cromosómica característica, el isocroma 12p (53). Por otra parte, no se ha demostrado un riesgo aumentado de inducción leucémica con el protocolo clásico de tratamiento PVB (cisplatino, bleomicina, vinblastina) (30).

De mayor preocupación para el futuro es la introducción de las epipodofilotoxinas en el arsenal terapéutico de estas neoplasias; en general, parecen demostrados algunos datos $(16,54)$ :

- La relación con la dosis acumulada de etopósido no está claramente establecida, aunque es probable que dosis totales mayores de $3000 \mathrm{mg} / \mathrm{m}^{2}$ (habitualmente en ciclos de 5 días de duración con dosis diarias de $200 \mathrm{mg} / \mathrm{m}^{2}$ ) estén asociados con un riesgo leucemogénico; el riesgo aumenta de forma importante a partir de dosis totales acumuladas mayores de 5.000 $\mathrm{mg} / \mathrm{m}^{2}$. Las dosis diarias de 100 a $120 \mathrm{mg} / \mathrm{m}^{2}$, con dosis totales acumuladas menores de $2.000 \mathrm{mg} / \mathrm{m}^{2}$, están asociadas con escaso poder leucemogénico.

- La forma de administración del fármaco tiene un papel fundamental en el riesgo de inducción leucémico, siendo las pautas de administración intermitentes intensivas en forma semanal o quincenal (utilizadas tradicionalmente en las leucemias agudas linfoblásticas infantiles) $(2,3)$ las de mayor poder leucemogénico, en comparación con las dosis continuas utilizadas en el tratamiento de los tumores sólidos en el adulto $(16,55)$.

No existen en la actualidad estudios que demuestren de forma fehaciente el posible efecto sinérgico entre el cisplatino y las epipodofilotoxinas en la inducción de neoplasias hematológicas (7).

\section{CÁNCER DE MAMA}

La asociación entre el tratamiento adyuvante del cáncer de mama y el riesgo de inducción de leucemias es bien conocido y se ha demostrado en varios estudios $(31,32)$; es probable que este riesgo sea sólo parcialmente achacable al tratamiento y exista una predisposición de dichas pacientes por el desarrollo de leucemias agudas. Clásicamente, el uso de tratamiento 
adyuvante con quimioterapia basada en agentes alquilantes del tipo melfalán y ciclofosfamida a dosis altas estaba asociado a un riesgo incrementado de inducción de leucemias, especialmente si se asociaba a técnicas de irradiación regional (32); de esta forma, la probabilidad estaba aumentada tanto con el uso de radioterapia aislada (RR 2,4), como con el uso de agentes alquilantes (RR 10) y con el uso combinado de radioterapia y quimioterapia (RR 17,4). El melfalán es claramente leucemogénico, presentando un riesgo diez veces superior al de la ciclofosfamida y una relación lineal entre la dosis total acumulada y el riesgo inductor (31). La ciclofosfamida a dosis totales superiores de $20 \mathrm{~g}$. presenta un riesgo de inducción de leucemias aumentado (RR 5,7), mientras que el uso de ciclofosfamida a dosis menores de $20 \mathrm{~g}$ no está asociado a dicho riesgo. El empleo de radioterapia regional a dosis elevadas estaba clásicamente asociado a un riesgo siete veces superior al de la población no tratada con irradiación.

En la actualidad, en el tratamiento adyuvante del cáncer de mama rara vez se utiliza el melfalán y la ciclofosfamida casi nunca alcanza dosis totales superiores a $20 \mathrm{~g}$; a su vez, la radioterapia regional se utiliza poco en la actualidad, prefiriéndose técnicas de irradiación más limitadas. Es por ello que, con los protocolos CMF (ciclofosfamida, metotrexate y 5-fluorouracilo), el riesgo inductor leucémico está solo discretamente aumentado, siendo 2,3 veces superior al de la población general (56). A su vez, la adición de antraciclinas no está asociado a un riesgo aumentado, al igual que el uso de la radioterapia locorregional.

Sin embargo, la quimioterapia a dosis altas en pacientes de mal pronóstico sí parece estar asociado a un aumento del riesgo leucemogénico, como cabría esperar con el uso de dosis elevadas de agentes alquilantes; estas combinaciones incluyen habitualmente dosis altas de ciclofosfamida asociadas a antraciclinas y otros agentes activos $(57,58)$. La combinación de antraciclinas y agentes alquilantes (incluyendo cisplatino) es probablemente leucemogénica; de forma característica, la leucemia secundaria que aparece es similar a la LAM secundaria a epipodofilotoxinas $(40,59)$. El riesgo asociado al empleo de antraciclinas en solitario es probablemente muy bajo, al igual que cuando se usan en aquellos protocolos que incluyen dosis bajas de ciclofosfamida y que no incluyen cisplatino.

Una asociación característica y descrita de forma aislada en la literatura médica es la existente entre el paclitaxel (utilizado en el cáncer de mama y de ovario) y una LAM secundaria característica (7) asociada a una alteración cromosómica propia, la inversión del cromosoma 16 -inv(16)(p13q22)-. El reconocimiento de este síndrome es importante, ya que parece existir una mejor respuesta de esta neoplasia al uso de arabinósido de citosina en forma de dosis escaladas, en comparación con el resto de LAM secundarias.

\section{TRASPLANTE DE MÉDULA ÓSEA Y DE CÉLULAS PROGENITORAS HEMATOPOYÉTICAS}

El uso tanto del trasplante autólogo y alogénico de médula ósea como del trasplante con células hematopoyéticas de sangre periférica en el tratamiento de las hemopatías malignas de mal pronóstico ha supuesto un avance significativo en la curación de estos pacientes. Sin embargo, dado que se basa en la quimioterapia a dosis altas como tratamiento de acondicionamiento y en técnicas de radioterapia como la irradiación corporal total, era lógico suponer que las LAM secundarias constituirían un importante problema en el pronóstico a largo plazo de estos pacientes. En efecto, los hallazgos mielodisplásicos en las médulas óseas de pacientes trasplantados son relativamente frecuentes $(60,61)$ y la probabilidad acumulada de estas leucemias en la literatura médica varía desde el $4 \%$ a los 5 años (62) hasta el $18 \%$ a los 6 años (63). En la actualidad está poco claro si dicho riesgo es secundario al uso de quimioterapia y radioterapia pretrasplante, a las técnicas de acondicionamiento y recogida de células hematopoyéticas, o como efecto acumulativo de todas esas exposiciones, aunque en la actualidad se tiende a considerar que el factor más importante es la quimioterapia pretrasplante (6,64-66). Clásicamente se ha considerado la irradiación corporal total como un factor de riesgo de inducción de leucemias (48), aunque diversos estudios recientes no han podido demostrar dicha asociación (64). Las técnicas de acondicionamiento y de recogida de células progenitoras hematopoyéticas con etopósido sí parecen estar relacionadas con un aumento del riesgo de inductor de leucemias con la traslocación característica 11q23 (64). Actualmente se considera que son factores de riesgo del huésped la edad avanzada en el momento del trasplante (67), el uso de radioterapia previa y la trombocitopenia en el momento del trasplante (63).

\section{PRONÓSTICO Y TRATAMIENTO}

Dada la relativa infrecuencia de los SMD y de las LAM secundarias y la ausencia de criterios diagnósticos definitorios de ambos procesos, existen pocos estudios donde se analicen específicamente sus factores pronósticos y, en muchos casos, esos datos se extrapolan de los trabajos realizados en los procesos primarios.

\section{SÍNDROMES MIELODISPLÁSICOS}

Entre los factores pronósticos de la supervivencia en los SMD primarios, parecen demostrados: el porcentaje de blastos en médula ósea, la clasificación FAB del proceso (siendo mejor para la anemia refractaria y progresivamente peor para síndromes más avanzados, como la AREB-t), la presencia de citopenias en sangre periférica y, sobre todo, la existencia de alteraciones citogenéticas características, como cariotipos complejos, trisomía del cromosoma 8 y cualquier anomalía del cromosoma $7(68,69)$. Estos tres factores (cariotipo, citopenias y porcentaje de blastos en médula ósea) han sido combinados en un sistema pronóstico (International Prognostic Scoring System) (Tabla V) que permite la clasificación de los síndromes mielodisplásicos en 4 grupos, evaluando la probabilidad de supervivencia a largo plazo y de conversión a LAM [70]. Dicha clasificación no es aplicable en un principio para los SMD secundarios, dada la dificultad de su clasificación de acuerdo a la FAB; sin embargo, la presencia de alteraciones citogenéticas consideradas desfavorables en prácticamente todos los casos y la frecuente conversión en LAM (con porcentajes que varían desde el 55 al 84\%) $(1,14,15)$, hacen de los SMD secundarios un proceso de mal pronóstico. Por tanto, para obtener la posible curación deben realizarse desde un principio terapéuticas agresivas.

El tratamiento de los SMD con quimioterapia intensiva (similar a la utilizada en la LAM) es controvertido y se basa 
TABLA V

SISTEM A PRO NÓSTICO INTERNACIONAL DE LOS SÍNDROM ES MIELODISPLÁSICOS

\begin{tabular}{lcc}
\hline Características & Valor & Puntuación (2) \\
\hline Blastos en médula & $<5$ & 0 \\
ósea (\%) & $5-10$ & 0,5 \\
& $11-20$ & 1,5 \\
& $21-30$ & 2,0
\end{tabular}

Cariotipo (1)

Buen pronóstico
Pronóstico intermedio
Mal pronóstico

\begin{tabular}{lll} 
Citopenias & $0-1$ & 0 \\
& $2-3$ & 0,5 \\
\hline
\end{tabular}

(1) Buen pronóstico: diploide, -4 , del (5q), del (20q); mal pronóstico: anomalías cromosoma 7 o complejas ( $\geq 3$ ); pronóstico intermedio: resto de anomalías. (2) Suma de puntuaciones: riesgo bajo $=0$; riesgo intermedio $1=0,5-1,0$; riesgo intermedio $2=1,5-2,5$; riesgo alto $=\geq 2,5$.

en el concepto teórico de que ambos procesos son una misma enfermedad, diferenciándose únicamente en el porcentaje de blastos en médula ósea. Por ejemplo, el grupo cooperativo Cancer and Leukemia Group B (CALGB) (71) trató 874 pacientes con LAM y 33 pacientes con SMD (de los subtipos AREB y AREB-t) con un esquema de quimioterapia utilizado habitualmente en la LAM; obtuvieron la remisión completa (RC) en el 79\% de los pacientes con SMD frente al $68 \%$ en los enfermos con leucemia aguda, con una duración de la RC de 11 frente a 15 meses y una supervivencia media de 13 frente a 16 meses, concluyendo que la diferenciación entre ambos procesos es de poco interés práctico desde el punto de vista terapéutico. Otros estudios apoyan estos hallazgos (72).

La combinación de arabinósido de citosina con una antraciclina es el pilar fundamental de la quimioterapia en estos procesos, con tasas de RC del 40 al $60 \%$ de los pacientes; sin embargo, la duración media de la RC y la supervivencia media suelen ser cortas, rara vez superiores a un año (10). La mortalidad durante la terapia de inducción varía desde el 5\% hasta el $20 \%$. Los porcentajes son aún peores en el caso de los procesos secundarios, con RC duraderas en menos del $10 \%$ de los pacientes $(4,10,73)$.

El uso de topotecán en monoterapia en pacientes pretratados presenta actividad en los SMD, con tasas de RC del $37 \%$ en casos de AREB y AREB-t y del $27 \%$ en pacientes con leucemia mielomonocítica crónica; la supervivencia media es de 10,5 meses, con una duración de la RC de 7.5 meses y mortalidad durante la terapia de inducción menor del $5 \%(74,75)$. Es probable que el uso combinado de topotecán y arabinósido de citosina constituya el pilar fundamental futuro de la quimioterapia en estos pacientes.

La única terapia curativa en la actualidad es el trasplante alogénico de médula ósea y debe considerarse el tratamiento de elección en estos pacientes, especialmente si son jóvenes y presentan factores de mal pronóstico de inicio (fundamentalmente las alteraciones citogenéticas características de los procesos secundarios) $(4,76)$. En la mayor parte de series, el trasplante alogénico se acompaña con una tasa de RC a largo plazo del $40 \%$, un $30 \%$ de recaídas y un $30 \%$ de muertes relacionadas con el trasplante; los mejores resultados se obtienen en pacien- tes con factores de buen pronóstico (fundamentalmente los subtipos anemia refractaria y anemia refractaria sideroblástica) $(77,78)$. Diversos estudios han demostrado que puede no ser necesaria la terapia de inducción previa al trasplante, especialmente en los SMD secundarios, dada la escasa respuesta en estos casos a las terapias de inducción $(4,76)$. A pesar de ser una opción atractiva, el trasplante autólogo de médula ósea debe considerarse una técnica experimental en la actualidad.

El uso de otros tratamientos, como los agentes diferenciadores (retinoides) y las citoquinas (eritopoyetina, factores estimuladores de colonias granulocíticas - G-CSF- o granulocitomacrofáficas -GM-CSF- y las interleukinas 3 y 6) permite habitualmente una mejoría de los parámetros hematológicos, aunque frecuentemente transitoria, y sólo está indicado como tratamiento único en aquellos casos de buen pronóstico (anemia refractaria simple o sideroblástica, de riesgo bajo o intermedio I), especialmente en pacientes de edad avanzada (79). El uso de otros agentes, como la amifostina, la azacitidina y la globulina antitimocítica, está actualmente en estudio, en particular en enfermos en que no sea posible la administración de quimioterapia clásica o la realización del trasplante de médula ósea (80).

\section{LEUCEMIA AGUDA MIELOIDE}

El desarrollo de una LAM secundaria es una complicación grave de la quimioterapia, que presenta escasas posibilidades de curación en la actualidad. Múltiples estudios han demostrado que el principal factor pronóstico para conseguir la curación de la enfermedad es la ausencia de alteraciones citogenéticas desfavorables $(5,81)$; en efecto, la $\mathrm{RC}$ a la quimioterapia sólo se da en el $13 \%$ de los pacientes con anormalidades del cromosoma 5 (del.5 o 5q-), independientemente de la presencia de anomalías en el cromosoma 7, en contraste con la respuesta en los pacientes con monosomía del cromosoma 7 o deleción del brazo largo (32\%), con cromosomas extranumerarios (43\%), o un cariotipo con otras anormalidades estructurales $(65 \%)$. La presencia de las alteraciones citogenéticas favorables descritas en la LAM primaria, como $t(15,17)$, $t(8,21)$ e inv(16) es rara en las leucemias secundarias (5). En general, la mayor parte de estudios ha demostrado una tasa escasa de respuestas en los pacientes con alteraciones de los cromosomas 5 y 7 , y tasas mayores en aquellos que presentan otras anormalidades cromosómicas. A pesar de ello, no existen pruebas actualmente para afirmar que la LAM secundaria a epipodofilotoxinas (con su traslocación característica 11q23) presente un pronóstico más benévolo que el de la LAM clásica o secundaria a agentes alquilantes $(2,3,16)$. Otros factores pronósticos desfavorables incluyen la edad avanzada en el momento del diagnóstico (>60 años), los antecedentes de mielodisplasia, la expresión del gen de resistencia multifarmacológica MDR1 y la presencia de blastos CD34+.

La terapéutica de inducción clásica de la LAM con una antraciclina (habitualmente idarrubicina) junto al arabinósido de citosina presenta una actividad escasa en las leucemias secundarias, alcanzándose RC sólo en el 50\% de los pacientes y habitualmente de corta duración (media de $<5$ meses) $(13,81)$. Este tratamiento sólo puede considerarse estándar en aquellos casos con alteraciones genéticas favorables, e incluso en estos casos los resultados son peores que en las leucemias primarias.

El trasplante alogénico es la única opción curativa disponible en la actualidad para los enfermos con LAM secunda- 
rias, aunque los resultados son también peores que en el caso de las primarias; se obtienen supervivencias a los 3 años del $25 \%$, frente al $45-60 \%$ en los procesos primarios $(4,81)$. Esta menor supervivencia parece deberse a un aumento de la mortalidad por el mayor número de recaídas y la mayor toxicidad del trasplante; son factores de peor pronóstico en estos pacientes la edad avanzada, el porcentaje de alteraciones citogenéticas, el número de blastos en médula ósea y la fibrosis de la médula, con la consiguiente menor reserva que favorece las muertes por toxicidad (4). A pesar de esos pobres resultados, el trasplante alogénico continúa siendo el tratamiento de elección (4-5, 81-83).

El uso de quimioterapia de inducción previo al trasplante no está claramente establecido: las tasas de RC son escasas (especialmente si hay alteraciones citogenéticas desfavorables), el riesgo de muerte durante la quimioterapia varía desde el 15 al $64 \%(4,81)$ (obviando a un porcentaje de pacientes del beneficio del trasplante) y no se ha podido demostrar que la realización del trasplante en $\mathrm{RC}$ mejore el pronóstico a largo plazo (4). Es por ello que en muchos centros no se emplea la quimioterapia de inducción previa al trasplante.

\section{Bibliografía}

1. Levine EG, Bloomfield CD. Leukemias and Myelodisplastic syndromes secondary to Drug, Radiation and Environmental Exposure. Semin Oncol 1992; 19: 47-84.

2. Pui CH, Ribeiro SC, Hancock ML, Rivera GK, Evans WE, Raimondi $\mathrm{SC}$, et al. Acute myeloid leukemia in children treated with epypodophillotoxins for acute lymphoblastic leukemia. N Engl J Med 1991; 325 : 1682-7.

3. Kreissman SG, Gelber RD, Cohen HG, et al. Incidence of secondary acute myelogenous leukemia after treatment of childhood acute lymphoblastic leukemia. Cancer 1992;70: 2208-13.

4. Anderson JE, Gooley TA, Schoch G, et al. Stem cell Transplantation for Secondary Acute Myeloid Leukemia: Evaluation of Transplantation as Initial therapy or Following Induction Chemotherapy. Blood 1997; 89: 2578-85.

5. de Witte T, Oosterveld M, Span B, et al. Stem cell transplantation for leukemias following myelodisplastic syndromes or secondary to cytotoxic therapy. Rev Clin Exp Hematol 2002; 6 (1): 72-85.

6. Sevilla J, Rodríguez A, Hernández-Maraver D, et al. Secondary acute myeloid leukemia and myelodisplasia after autologous peripheral blood progenitor cell transplantation. Ann Hematol 2002; 81 (1): 11-5.

7. Smith MA, McCaffrey RP, Karp JE. Secondary Leukemias: Challenges and Research Directions. J Natl Cancer Inst 1996; 88: 407-18.

8. Head DR. Revised classification of acute myeloid leukemia. Leukemia 1996; 10: 1826-31

9. Paietta E. Classification of acute leukemias: Proposals for the immunolgical classification of acute leukemias. Leukemia 1995; 9; 2147-8.

10. Cheson BD. The Myelodisplastic Syndromes. Oncologist 1997; 2 (1): 28-39.

11. Bennet JM, Catovsky MT, Daniel MT, et al. The French-American-British (FAB) Cooperative Group: Proposals for the classification of myelodisplastic syndromes. Br J Haematol 1982; 51: 189-99.

12. Rigolin GM, Cureo A, Roberti MG, et al. Exposure to myelotoxic agents and myeldisplasia: case-control study and correlation with clinicobiological findings. Br J Haematol 1998; 103 (1); 189-97.

13. Thirman MJ, Larson RA. Therapy-related myeloid leukemia. Hematol Oncol Clin North Am 1996; 10: 293-320.

14. Boogaerts MA, Verhoef GF, Demuynck H. Treatment and prognostic factors in myelodisplastic syndromes. Baillieres Clin Haematol 1996; 9: 161-83.

15. Kouides PA, Bennett JM. Understanding the Myelodisplastic Syndromes. Oncologist 1997; 2: 389-401.

\section{CONCLUSIONES}

La aparición de neoplasias secundarias (sólidas o hematológicas) es la complicación más grave que pueden presentar los pacientes que sobreviven al cáncer. Entre ellas destacan las LAM y SMD asociados al empleo de alquilantes, epipodofilotoxinas, radioterapia sobre campos extensos y, en menor medida, taxanos. Se desconoce su incidencia real debido a la diversidad de pautas terapéuticas y al diferente pronóstico de los tumores primarios. El diagnóstico se basa en la sospecha clínica y en la presencia de alteraciones citogenéticas compatibles, pero el pronóstico es uniformemente desfavorable. La terapéutica se basa en el uso de arabinósido de citosina, idarubicina y, más recientemente, topotecán. El trasplante alogénico parece ser la opción más eficaz pero es especialmente tóxico en enfermos muy pretratados. Por todo ello y, dado el aumento en la supervivencia de algunos tumores sólidos, se hace necesario un seguimiento a largo plazo de estos enfermos y una optimización de las pautas terapéuticas empleadas en la actualidad para minimizar la incidencia de LAM y SMD secundarios.
16. Smith MA, Rubinstein L, Anderson JL, et al. Secondary leukemia or myelodisplastic syndrome after treatment with epypodophillotoxins. J Clin Oncol 1999; 17: 569-77.

17. Kantarjian HM, Keating MJ, Walters RS, et al. Therapy-related leukemia and myelodisplastic syndrome: clinical, cytogenetic and prognostic features. J Clin Oncol 1986; 4: 1748-57.

18. Wilson CS, Traweek T, Slovak ML, et al. Myelodisplastic syndrome occurring after autologous bone marrow transplantation for lymphoma: morphologic features. Am J Clin Pathol 1997; 108: 369-77.

19. Michels SD, McKenna RW, Arthur DC, et al. Therapy-related acute myeloid leukemia and myelodisplastic syndromes: a clinical and morphological study of 65 cases. Blood 1985; 65: 1364-72.

20. Pagano L, Pulsoni A, Tosti ME, Annino L, Mele A, Camera A, et al Acute lymphoblastic leukaemia occurring as second malignancy: report of the GIMEMA Archive of Adult Acute Leukaemia. Br J Haematol 1999; 106: 1037-40.

21. Beaty O, Hudson MM, Greenwald C, Lua X, Fang L, Wilimas JA, et al. Subsequent malignancies in children and adolescents after treatment for Hodgkin's Disease. J Clin Oncol 1995; 13: 603-9.

22. de Greef GE, Hagemeijer A. Molecular and cytogenetic abnormalities in acute myeloid leukemia and myelodosplastic syndromes. Ballieres Clin Haematol 1996; 9: 1-18.

23. Johansson B, Mertens F, Heims S, et al. Cytogenetics of secondary myelodisplasia (sMDS) and acute nonlymphocytic leukemia (sANLL). Eur J Haematol 1991; 47: 17-27.

24. Pedersen-Bjergaard J, Philip P, Larsen SO, et al. Chromosome aberrations and prognostic factors in therapy-related myelodisplasia and acute nonlymphocytic leukemia. Blood 1990; 76: 1083-91.

25. van Leeuwen FE, Chorus AM, van den Belt-Dusebout AW, et al. Leukemia risk following Hodgkin's disease: relation to cumulative dose of alkylating agents, treatment with teniposide combinations, number of episodes of chemotherapy, and bone marrow damage. J Clin Oncol 1994; 12: 1063-73.

26. Travis LB, Holowaty EJ, Bergfeldt K, Lynch CF, Kohler BA, Wiklund $\mathrm{T}$, et al. Risk of leukemia after platinum-based chemotherapy for ovarian cancer. N Engl J Med 1999; 340: 351-7.

27. Greene MH, Harris EL, Gerhenson DM, et al. Melphalan may be a more potent leukemogen than cyclophosphamide. Ann Inter Med 1986; 105: 360-7.

28. Cuzick J, Erskine S, Edelman D. A comparison of the incidence of the myelodisplastic syndrome and acute myeloid leukemia following melp 
halan and cyclophosphamide treatment for myelomatosis. Br J Cancer 1987; 55: 523-9.

29. Stott H, Fox W, Girling DJ, et al. Acute leukemia after busulphan. BMJ 1977; 2: 1513-7.

30. Roth BJ, Greist A, Kubilis PS, Williams SD. Cisplatin-based combination chemotherapy for disseminated germ cell tumours: long-term follow-up. J Clin Oncol 1988; 6: 1239-47.

31. Curtis RE, Boice JD, Stovall M, et al. Risk of leukemia after chemotherapy and radiation treatment for breast cancer. N Eng J Med 1992; 326: 1745-51.

32. Fisher B, Rockette H, Fisher ER, et al. Leukemia in breast cancer patients following adjuvant chemotherapy or postoperative radiation: The NSABP experience. J Clin Oncol 1985; 3: 1640-58.

33. Andrieu JM, Ifran N, Payen C, Fermanian J, Coscas Y, Flandrin G. Increased risk of acute nonlymphocitic leukemia after extended-field radiation therapy combined with MOPP chemotherapy for Hodgkin's disease. J Clin Oncol 1990; 8: 1148-54.

34. van Leeuwen FE, Klokman WJ, Hagenbeek A, et al. Second cancer risk following Hodgkin's disease: a 20-year follow-up study. J Clin Oncol 1994: 12: 312-25.

35. Sandoval C, Pui CH, Bownan LC, Heaton D, Hurwitz CA, Raimondi $\mathrm{SC}$ et al. Secondary acute myeloid leukemia in children previosly treated with alkylating agents, intercalating topoisomerase II inhibitors and irradiation. J Clin Oncol 1993; 11: 1039-45.

36. Archimbaud E, Charrin C, Magaud JP, Campos L, Thomas X, Fiere D, et al. Clinical and biological characteristics of adult de novo and secondary acute myeloid leukemia with balanced 11q23 chromosomal anomaly or MLL gene rearrangement compared to cases with unbalanced 11q23 anomaly: confirmation of the existence of different entities with $11 \mathrm{q} 23$ breakpoint. Leukemia 1998; 12: 25-33.

37. Pedersen-Bjergaard J, Andersen MK, Johansson B. Balanced chromosome aberrations in leukemias following chemotherapy with DNAtopoisomerase II inhibitors. J Clin Oncol 1998; 16: 1897-8.

38. Karp J, Smith M. Modifying risks of secondary leukemias: is drug scheduling important? [comment]. J Natl Cancer Inst 1996; 88: 1787-9.

39. Chen C-L, Fuscoe J, Liu Q, et al. Relationship between cytotoxicity and site-specific DNA recombination after in vitro exposure of leukemia cells to etoposide. J Natl Cancer Inst 1996; 88: 1840-7.

40. Carli PM, Sgro C, Parchin-Geneste N, Isambert N, Mugneret F, Girodon F, et al. Increased therapy-related leukemia secondary to breast cancer. Leukemia 2000; 14: 1014-7.

41. Swerdlow AJ, Douglas AJ, Hudson GV, Hudson BV, Bennett MH, MacLennan KA. Risk of second primary cancers after Hodgkin's disease by type of treatment: analysis of 2846 patients in the British National Lymphoma Investigation. Br Med J 1992; 304: 1137-43.

42. Pedersen-Bjergaard J, Specht L, Larsen SO, et al. Risk of therapy-related leukemia and preleukemia after Hodgkin's disease: relation to age, cumulative dose of alkylating agents, and time from chemotherapy. Lancet 1987; $2: 838$.

43. Abrahamsen JF, Andersen A, Hannisdal E, et al. Second malignancies after treatment of Hodgkin's disease: The influence of treatment, follow-up and age. J clin Oncol 1993; 11: 255-61.

44. Swerdlow AJ, Douglas AJ, Vaughan Hudson B, et al. Risk of second primary cancer after Hodgkin's disease in the British National Lymphoma Investigation: relationships to host factors, histology and stage of Hodgkin's disease, and splenectomy. Br J Cancer 1993; 68: 1006-11.

45. Travis LB, Curtis RE, Glimelius B, et al. Second cancers among longterm survivors of non-Hodgkin's lymphoma. J Natl Cancer Inst 1993; 85: 1932-7.

46. Pedersen-Bjergaard J, Ersboll J, Sorensen HM, et al. Risk of acute nonlymphocytic leukemia and preleukemia in patients treated with cyclophosphamide for non-Hodgkin's lymphomas. Comparisons with results obtained in patients treated for Hodgkin's disease and ovarian carcinoma with other alkylating agents. Ann Inter Med 1985; 103: 195-200.

47. Travis LB, Weeks J, Curtis RE, et al. Leukemia following low-dose total body irradiation and chemotherapy for non-Hodgkin's lymphoma. J Clin Oncol 1996; 14: 565-71.

48. Travis LB, Curtis RE, Stovall M, et al. Risk of leukemia after treatment for non-Hodgkin's lymphoma. J Natl Cancer Inst 1994; 86: 1450-7.

49. Kaldor JM, Day NE, Petterson F, et al. Leukemia following chemotherapy for ovarian cancer. N Engl J Med 1990; 322: 1-6

50. Boshoff C, Begent RH, Oliver RT, et al. Secondary tumours following etoposide-containing therapy for germ cell cancer. Ann Oncol 1995; 6: $35-43$.
51. van Leeuwen FE, Stiggelbout AM, van den Belt-Dusebout AW, et al. Second cancer risk following testicular cancer. A follow-up study of 1.909 patients. J Clin Oncol 1993; 11: 415-24.

52. Bokemeyer C, Schmoll H-J. Treatment of testicular cancer and the development of secondary malignancies. J Clin Oncol 1995; 13: 283-92.

53. Nichols CR, Roth BJ, Heerema N, et al. Hematologic neoplasia associated with primary mediastinal germ-cell tumours. N Engl J Med 1990; 322: 1425-9.

54. Smith M, Rubinstein L, Cazenave L et al Report of the Cancer Therapy Evaluation Program (CTEP) monitoring plan for secondary acute myeloid leukemia following treatment with epypodophillotoxins. J Natl Cancer Inst 1993; 85: 554-58.

55. Relling MV, Yanishevski, Nemec J, Evans WE, Boyett JM, Behm FG, et al. Etoposide and antimetabolite pharmacology in patients who develop secondary acute myeloid leukemia. Leukemia 1998; 12: 346-52.

56. Valagussa P, Moliterni A, Terenziani M, Zambetti M, Bonnadonna G. Second malignancies following CMF-based adjuvant chemotherapy in resectable breast cancer. Ann Oncol 1994; 5: 803-8.

57. Laughlin MJ, McGaughey DS, Crews JR, et al. Secondary myelodisplasia and acute leukemia in breast cancer patients after autologous bone marrow transplant. J Clin Oncol 1998; 16: 1008-12.

58. Roman-Unfer S, Bitran JD, Hanauer S, et al. Acute myeloid leukemia and myelodisplasia following intensive chemotherapy for breast cancer. Bone Marrow Transplant 1995; 16: 163-8.

59. Pedersen-Bjergaard J, Sigsgaard TC, Nielsen D, et al. Acute monocytic or myelomonocytic leukemia with balanced chromosome translocations to band $11 \mathrm{q} 23$ after therapy with 4-epi-doxorrubicin and cisplatin or cyclophosphamide for breast cancer. J Clin Oncol 1992; 10: 1444-51.

60. Stone RM. Myelodisplastic syndrome after autologous trasplantation for lymphoma: the price of progress? Blood 1994; 83: 3437-40.

61. Sobecks RM, Le Beau MM, Anastasi J, et al. Myelodisplasia and acute leukemia following high-dose chemotherapy and autologous bone marrow or peripheral blood stem cell transplantation. Bone Marrow Transpl 1999, 23: 1161-5.

62. Darrington DL, Vose JM, Anderson JR, et al. Incidence and characterization of secondary myelodisplastic syndrome and acute myelogenous leukemia following high-dose chemoradiotherapy and autologous stem cell trasplantation for lymphoid malignancies. J Clin Oncol 1994; 12 : 2527-34.

63. Stone RM, Seuberg D, Saiffer R, et al. Myelodisplastic syndrome as a late complication following autologous bone marrow trasplantation for non-Hodgkin's lymphoma. J Clin Oncol 1994; 12: 2535-42.

64. Krishnan A, Bhatia S, Slovak ML, Arber DA, Niland JC, Nademanee A, et al. Predictors of therapy-related leukemia and myelodisplasia following autologous transplantation for lymphoma: an assesment of risk factors. Blood 2000; 95: 1588-93.

65. Pedersen-Bjergaard J, Pedersen M, Myhre J, et al. High risk of therapyrelated leukemia after BEAM chemotherapy and autologous stem cell transplantation for previosly treated lymphomas is mainly related to primary chemotherapy and not to the BEAM transplantation procedure. Leukemia 1997, 11: 1654-60.

66. Harrison CN, Gregory W, Hudson GV, et al. High-dose BEAM chemotherapy with autologous hematopoietic stem cell transplantation for Hodgkin's disease is unlikely to be associated with a major increased risk of secondary MDS/AML. Br J Cancer 1999; 81: 476-83.

67. Bhatia S, Ramsay NKC, Steinbuch M, et al. Malignant neoplasms following bone marrow transplantation. Blood 1996; 87: 3633-9.

68. Sanz GF, Sanz MA, Vallespí, et al. Two regression models and a scoring system for predicting survival and planning treatment in myelodisplastic syndromes: a multivariate analysis of prognostic factors in 370 patients. Blood 1989; 74: 395-408.

69. Hiddemann W, Jahns-Streubel G, Verbeek W, et al. Intensive therapy for high-risk myelodisplastic syndromes and the biological significance of karyotype abnormalities. Leuk Res 1998; S23: 23-6.

70. Greenberg P, Cox C, Lebeau MM, et al. International scoring system for evaluating prognosis in myelodisplastic syndromes. Blood 1997; 89: 2079-88.

71. Bernstein SH, Brunetta VL, Davey FR, et al. Acute myeloid leukemia-type chemotherapy for newly-diagnosed patients with antecedent cytopenias having myelodisplastic syndrome as defined by FrenchAmerican-British criteria: a Cancer and Leukemia Group B study. J Clin Oncol 1996; 14: 2486-94.

72. Estey HE, Thall P, Beran M, et al. Effect of diagnosis (refractory anemia with excess blasts, refractory anemia with excess blasts in transformation, or acute myeloid leukemia [AML]) on outcome of AMLtype chemotherapy. Blood 1997; 90: 2969-77. 
73. Estey EH, Kantarjian HM, O'Brien S, et al. High remission rate, short remission duration in patients with refractory anemia with excess blasts (RAEB) in transformation (RAEB-t) given acute myelogenous leukemia (AML)-type chemotherapy in combination with granulocyte-CSF (G-CSF). Cytojines Mol Ther 1995; 1: 21-8.

74. Beran M, Kantarjian H, O’Brien S, et al. Topotecan, a topoisomerase I inhibitor, is active in the treatment of myelodisplastic syndrome and chronic myelomonocitic leukemia. Blood 1996; 88: 2473-9.

75. Beran M, Estey E, O’Brien SM, et al. Results of topotecan single-agent therapy in patients with myelodisplastic syndromes and chronic myelomonocytic leukemia. Leuk Lymph 1998;31(5-6): 521-31.

76. Runde V, deWitte T, Arnold R, et al. Bone marrow trasplantation from HLA-identical siblings as first-line treatment in patients with myelodisplastic syndromes: early transplantation is associated with improved outcome. Bone Marrow Transplant 1998; 21: 255-61.

77. Appelbaum FR, Anderson J. Allogeneic bone marrow transplantation for myelodysplastic syndrome: outcomes analysis according to IPSS Leukemia 1998;12 (Supl. 1): S25-S29.
78. Ballen KK, Gilliland DG, Guinan EC, et al. Bone marrow transplantation for therapy-related myelodisplasia: Comparison with primary myelodisplasia. Bone Marrow Transpl 1997; 20: 737-43.

79. Musto P, Falcone A, Carotenuto M, et al. Granulocyte colony-stimulating factor and erythropoietin for anemia of myelodysplastic syndromes: a real improvement with respect to erythropoietin alone?. Blood 1994; 84: 1687-8.

80. Cheson BD, Zweibel JA, Dancey J, et al. Novel therapeutic agents for the treatment of Myelodisplatic Syndromes. Semin Oncol 2000; 27: 560-77.

81. Witherspoon RP, Deeg HJ, Storer B, et al. Hematopoietic stem-cell transplantation for treatment-related leukemia or myelodisplasia. J Clin Oncol 2001; 19: 2134-41.

82. De Witte T, Gratwohl A. Bone marrow transplantation for myelodisplastic syndromes and secondary leukemias. Br J Hematol 1993; 84: 361-4.

83. Longmore G, Guinan EC, Weinstein HJ, et al. Bone marrow transplantation for myelodisplasia and secondary acute nonlymphoblastic leukemia. J Clin Oncol 1990; 8: 1707-14. 